\title{
Gradhiva
}

\section{Daniel Fabre, Bataille à Lascaux. Comment l'art préhistorique apparut aux enfants}

Paris, L'Échoppe, 2014

\section{Rémi Labrusse}

\section{OpenEdition}

\section{Journals}

Édition électronique

URL : http://journals.openedition.org/gradhiva/3119

DOI : $10.4000 /$ gradhiva. 3119

ISSN : 1760-849X

\section{Éditeur}

Musée du quai Branly Jacques Chirac

\section{Édition imprimée}

Date de publication : 1 octobre 2015

Pagination : 238-240

ISBN : 978-2-35744-092-0

ISSN : 0764-8928

\section{Référence électronique}

Rémi Labrusse, "Daniel Fabre, Bataille à Lascaux. Comment l'art préhistorique apparut aux enfants », Gradhiva [En ligne], 22 | 2015, mis en ligne le 18 septembre 2015, consulté le 22 septembre 2020. URL http://journals.openedition.org/gradhiva/3119; DOI : https://doi.org/10.4000/gradhiva.3119

Ce document a été généré automatiquement le 22 septembre 2020.

(c) musée du quai Branly 


\section{Daniel Fabre, Bataille à Lascaux.} Comment l'art préhistorique apparut aux enfants

Paris, L’Échoppe, 2014

Rémi Labrusse

\section{RÉFÉRENCE}

Daniel Fabre, Bataille à Lascaux. Comment l'art préhistorique apparut aux enfants. Paris, L'Échoppe, 2014, 142 p. 
DANIEL FABRE

\section{Bataille à Lascaux}

Comment l'art préhistorique apparut aux enfants

L'ÉCHOPPE

On se tromperait si on allait chercher dans le livre de Daniel Fabre un nouveau maillon dans le chapelet de l'hagiographie bataillienne, articulée en l'occurrence au commentaire de Lascaux et la naissance de l'art, le grand livre que Georges Bataille a consacré en 1955 à la plus célèbre des grottes ornées paléolithiques, découverte en 1940 par quatre adolescents, ouverte au public en 1948, irrémédiablement endommagée par l'usage touristique et finalement refermée en 1963. Non pas qu'il s'agisse pour l'auteur de déconstruire ni même de mettre à distance la pensée de Bataille : au contraire, la dimension poétique de cette pensée suscite à tout moment son admiration. Qu'est-ce que le poétique dans la pensée? La fatalité d'une réflexion conceptuelle à être indissolublement une épreuve existentielle, à s'enraciner dans un amont des mots qui les trouble et les enflamme, qui les éclaire d'en dessous. Et quelle est la pensée ou plutôt «l'énigme» qui se formule ainsi poétiquement chez Bataille, depuis toujours? Celle «d'un sacré immanent qui confronte l'humanité au mystère de son propre engendrement»(p. 69), ce dont, quelques années avant de mourir, il a découvert l'attestation la plus éclatante à Lascaux. Lui qui aimait s'identifier à ceux qui, « comme des mioches cherchant les fentes d'une palissade, tâchent de regarder par les failles de ce monde » (cité p. 73), s'est laissé affecter intimement par cette énigme, cherchant à conserver intactes dans son questionnement la palpitation et, aussi bien, la violence de l'enfance. Du coup, la pensée qu'il élabore à Lascaux est aussi une épreuve où, physiquement et matériellement, se conjoignent le singulier et l'universel, son destin personnel, remontant à sa propre enfance, et celui de l'humanité comme telle. Du coup, également, il en vient à formuler comme nul autre avant lui - et à nous rendre pathétiquement sensible - un des événements majeurs de la modernité : l'invention de la préhistoire.

2 Voilà pourquoi le livre de Fabre n'est pas d'abord une exégèse bataillienne : s'il est cela aussi, s'il la développe même avec une sorte d'empathie, c'est pour se diriger au-delà, en direction de l'idée préhistorique - construction mentale, grand réseau de 
représentations qu'a passionnément habité (et que continue d'habiter) la conscience moderne, avec en son cœur l'art dit " préhistorique ». Bataille à Lascaux est un livre sur l'incandescence spécifique de l'idée préhistorique pour nous aujourd'hui, sur les conditions de possibilité de son émergence, sur les désirs et les manques qui l'ont fait naître et sur ce qu'elle est allée toucher de plus profond, de plus tragique en nous. La constatation de départ, c'est que le tremblement personnel éprouvé par Bataille au contact de l'art pariétal magdalénien est ce qui confère secrètement sa puissance à son discours philosophique et esthétique sur la préhistoire - y compris lorsque ce discours se brise et que le silence y succède à l'effort herméneutique. À partir de là, logiquement, Fabre a pris la décision méthodologique de rendre inséparables deux enquêtes : l'une enracinée dans le singulier, sur les conditions de possibilité de l'œuvre de Bataille, pourchassées jusqu'aux tréfonds de sa biographie ; l'autre, d'anthropologie culturelle, qui - pour reprendre les termes de l'auteur - s'attache à " saisir le réseau de connexions culturelles qui a rendue possible et pensable, hic et nunc, après des millénaires d'invisibilité, la réalité de l'art pariétal » (p. 85).

Comme toute enquête, ces deux-là mènent à des révélations, éminemment convaincantes, dont on suit le dévoilement progressif page après page et qu'il serait regrettable de dévoiler ici de façon forcément sommaire et maladroite. Il suffira de dire que l'impeccable rigueur des raisonnements et la vastitude de l'érudition font qu'on apprend beaucoup dans ce petit livre, et surtout beaucoup de choses inattendues : par exemple, sur l'inceste comme point de rencontre entre Proust, Bataille et les peintres magdaléniens; ou sur la similitude des expériences préhistoriques vécues et retranscrites par les nombreux prêtres découvreurs de grottes au début du xxe siècle et, à l'autre bout du spectre idéologique français, par un député communiste d'ascendance protestante comme Paul Vaillant-Couturier. D'autres aspects étaient connus mais traités anecdotiquement, jusqu'à ce que Fabre s'en empare : le fait, en particulier, que les enfants ou les adolescents ont joué un rôle majeur dans les découvertes de grottes ornées paléolithiques jusqu'à Lascaux ; ou que la grotte, en tant que telle, a été un site privilégié pour les apparitions mariales qui se multiplient au même moment que l'invention de la préhistoire, à partir des années 1850, jusqu'à produire une situation de rivalité, sur le site de Lourdes, entre les aménagements dévotionnels (la grotte de Massabielle, où la jeune Bernadette Soubirous a vu la Vierge) et l'étude scientifique des traces laissées aux mêmes endroits par les hommes du paléolithique supérieur (la grotte des Espélugues, juste au-dessus). On se doutait bien que tout cela avait maille à partir avec les résurgences erratiques d'un sacré primordial, déchirant les données de l'histoire et de la culture pour contrebalancer les apories métaphysiques et, aussi bien, les exclusions sociales d'une société industrielle et bourgeoise sécularisée. Mais jamais les liens entre les apparitions de la Vierge et les inventions (comme on dit en archéologie) de grottes paléolithiques n'avaient été élaborés dans le contexte systématique d'une anthropologie de la modernité comme le fait l'auteur de Bataille à Lascaux. Jamais non plus l'action des enfants n'avait été vraiment menée au-delà d'une réflexion socio-historique sur le rôle de l'amateurisme local dans les pratiques d'archéologie préhistorique et dans la constitution même de la discipline préhistorienne. Fabre, s'appuyant sur Bataille, en fait, quant à lui, l'indice que «les enfants » étaient directement réceptifs à un sacré sauvage que l'Occident moderne est allé puiser aux sources paléolithiques, tout en s'affairant contradictoirement à le recouvrir, à le refouler sous les couches du positivisme archéologique, du nationalisme patrimonial et du divertissement touristique. 
4 Aucune démarche, de fait, n'est plus adaptée que celle de l'anthropologie pour explorer les données constitutives de ce phénomène majeur qu'est l'invention de la préhistoire, et qui ne fait qu'un avec ce qu'on nomme au même moment la modernité - pour autant qu'on y associe les méthodes de l'histoire culturelle et de la psychologie, comme le fait Fabre. Car ce qui s'exprime dans ce surgissement de l'évidence "préhistorique », en 1860 , est un besoin de sortie hors du relativisme inhérent à une conception historicisée du monde et, a contrario, de dégagement d'un socle anthropologique où se dessine une figure immuable de l'humain. Cet anthropologisme panique qui irrigue, depuis l'origine, la pensée du préhistorique, en deçà des procédures scientifiques, comment le comprendre mieux qu'avec les instruments de l'anthropologie même? C'est elle qui permet de saisir la tension intellectuelle fondamentale par laquelle la science historique, en parvenant à son accomplissement dans la prise en compte de la haute antiquité de l'homme, se retourne en fait souterrainement contre elle-même et met au jour la relativité de son propre relativisme.

5 Plus que tout autre témoignage des périodes paléolithiques, l'art pariétal, à partir de la toute fin du XIXe siècle, a incarné physiquement ce renversement de la vision évolutive et contextualisée de l'homme : non seulement parce que sa datation absolue est restée très longtemps impossible (et est encore souvent hésitante tant que l'absence de matériaux organiques comme le charbon de bois exclut le recours au carbone 14); mais aussi parce que le milieu souterrain, en tant que tel, est caractérisé par une double impression d'éternité (l'obscurité protectrice, la température stable) et de fraîcheur étrangement présente (l'humidité intense, le bruit des gouttes, les concrétions en constante formation, la boue indéfiniment malléable); enfin et surtout, parce que les traces humaines posées sur les parois laissent tout récit, toute histoire à l'état virtuel, flottant, dissous, et, du coup, par leur présence muette, invitent le visiteur à une sorte de vague abandon fusionnel - « une étrange sensation de douceur animale touchant à la métempsychose ", écrit Bataille (cité p. 94). Lascaux, en 1940, a soudain constitué le spectacle éclatant de cette concaténation des dimensions temporelles, alors que la plupart des autres grottes réclamaient un œil aiguisé pour débrouiller les enchevêtrements de traits, le plus souvent médiatisés par des relevés qui transformaient leur matérialité touffue en pure image. Mais dans tous les cas, il s'agit bien, pour l'homme du xxe siècle, de s'immerger concrètement, à tort ou à raison, dans de l'immémorial : la présence de l'homme est là absolument, elle ne dit rien d'autre qu'elle-même, elle échappe à toute construction narrative stable; le passé le plus vertigineux y apparaît (Fabre parle d'un besoin général de "révélations ») intégralement présent. Notons que, bien après Lascaux, cette soif d'immanence préhistorique est pour beaucoup dans la fascination exercée par la grotte Chauvet, découverte en 1994 près du Pont-d'Arc, en Ardèche : là, plus sans doute que nulle part ailleurs, le temps se trouve comme transpercé par l'intacte présence matérielle des moindres tressautements de gestes humains et animaux accomplis il y a des dizaines de milliers d'années, et c'est ce transpercement même que la République conserve désormais religieusement, comme la cella interdite d'un temple où s'actualise un même besoin de faire éclater le noyau du temps, c'est-à-dire une même soif de sacré, des chasseurs cueilleurs aurignaciens aux scientifiques et aux gardiens modernes du patrimoine.

6 Le phénomène n'est évidemment pas simple: comme le montre aussi Fabre, les «sciences positives» (dont la science préhistorique) n'ont eu de cesse de parvenir à 
circonscrire, sinon à étouffer «l'illimitation métaphysique de leur objet » (p. 110), en en établissant la chronologie, donc l'historicité, en densifiant le contenu documentaire des données archéologiques, en réfutant (jusqu'à la violence) les interprétations trop affirmatives. Mais précisément, ce contraste entre la surabondance des observations et la fragilité ou la pauvreté volontaire des interprétations a produit un vide dans lequel se sont engouffrées les exigences du rêve : ces rêves qui donnent aussi sa vérité, in fine, à l'idée préhistorique et que Bataille fut le seul, selon Fabre, à prendre en compte avec autant de rigueur analytique que d'élan lyrique.

7 D'un côté, Bataille le savant refuse de céder à la «légende dorée » (p. 36) de la préhistoire, sensible dans l'imagerie populaire dès le xix ${ }^{e}$ siècle et régulièrement réactualisée par les pulsions fantaisistes d'écrivains ou même de spécialistes - que ce soit André Malraux présentant Lascaux dans ses Antimémoires comme une cache d'armes où il se serait retrouvé par surprise en 1944 (p. 12), Vaillant-Couturier se décrivant dans ses souvenirs d'enfance en train de découvrir un improbable burin bleu turquoise dans une niche reculée de la grotte ariégeoise du Mas-d'Azil (p. 84), ou l'abbé Breuil lui-même, préhistorien majeur et dominant, célébrant sa « dame blanche » du Brandberg (en fait la figure d'un jeune chasseur peinte sur un rocher de Namibie) comme une "très ancienne jeune fille » dont «il a parlé au monde des vivants après avoir rêvé à ses pieds de l'infini mystère de l'histoire " (cité p. 68). D'un autre côté, Bataille le poète (c'est-à-dire identiquement le penseur) se met à l'écoute d'une résonance profonde qui irrigue tous ces fantasmes agrégés autour de l'idée préhistorique; la trouve à l'état natif, en quelque sorte, dans les récits des jeunes découvreurs, en particulier Jacques Marsal et Marcel Ravidat avec lesquels il s'entretient longuement; et en dégage une vérité anthropologique à laquelle l'attache personnellement, sur un plan non pas magique mais poétique, sa propre sensibilité. Il est à l'écoute de la joie surnaturelle des inventeurs, jeunes et moins jeunes, gamins courant les chemins et prêtres en quête de ce que la terre recèle de surnaturel; il éprouve à son tour ce sentiment d'extase, en effaçant, autant que faire se peut, la perception des aménagements récents qui rendent la descente dans la grotte pareille à un couloir de métro (cité p. 31); enfin, il relie cette expérience fondatrice à des intuitions qu'il a déjà partagées avec ses amis des avant-gardes parisiennes, au cours des années 1930, lorsque l'idée préhistorique entrait en fusion avec une poétique de l'apocalypse, au sens étymologique du terme : dévoilement de l'absolu produit par une rupture avec la temporalité, «simple élimination du facteur temps ", comme l'écrivait Brassaï («Du mur des cavernes au mur d'usine », Minotaure, n 3-4, 1933, p. 6), ou «télépathie archaïsante " grâce à laquelle «la fin rejoint le commencement ", selon Carl Einstein - qui commentait en l'occurrence les collages de Miró, manifestement hantés par «l'âge des cavernes ", dans la revue même de Bataille, Documents, en 1930 (no 4, p. 243).

8 Au total, donc, ce que Bataille met au jour activement, c'est l'entrechoquement des contraires que produit la pensée de la "préhistoire » et où surgit, comme l'étincelle au contact des pierres, une sorte de béance métaphysique : choc entre l'attestation du très ancien et la sensation du très actuel; entre le sentiment de totale historicité et l'intuition d'un inexplicable déchirement de cette historicité même; enfin, entre l'impression d'identité, de confraternité charnelle avec l'homme du paléolithique et celle, tout au contraire, d'altérité radicale, de plongée soudaine dans un monde radicalement autre qui ne donne à voir - et avec quelle intensité - que l'évidence de son impénétrable secret. Là encore, la récurrence des réactions lors de l'invention des 
grottes ornées est caractéristique d'une constante que l'anthropologie est à même d'éclairer : "Ils sont venus après les grands ours ", écrivait le jeune Max Begouën, un des trois frères Begouën, au géographe Jean Brunhes, en 1912, après avoir découvert la grotte du Tuc d'Audoubert (cité p. 43); « Nous avons l'impression d'avoir pénétré dans un autre monde» (cité p. 44), disait de son côté André David, lui aussi adolescent, responsable de la découverte de la partie ornée de la grotte du Pech Merle; et ajoutons le "Ils sont venus" prononcé à son tour en 1994 par Éliette Brunel-Deschamps, inventrice de la grotte Chauvet-Pont-d'Arc avec Jean-Marie Chauvet et Christian Hillaire, lorsque lui sont apparus, dans la galerie dite du Cactus, un premier tracé de quelques traits à l'ocre puis un petit mammouth (spéléologue chevronnée comme ses compagnons, elle retrouvait ainsi spontanément l'émerveillement de l'enfance dans ces trois mots en passe de devenir, pour cette raison même, emblématiques). Ce «Ils » ne désigne certes pas des êtres d'un " autre monde "; mais il n'en doit pas moins être pris très au sérieux, aurait dit Bataille, puisqu'il marque un sentiment intérieur de l'altérité à soi comme condition métaphysique et tragique de l'homme. De cette condition, la modernité veut ouvertement s'affranchir et pourtant, obscurément, elle en porte sans fin le deuil: ce pourquoi elle invente l'idée préhistorique, nœud flamboyant de contradictions, avant d'inventer les grottes ornées elles-mêmes.

Fabre a montré comment l'anthropologie préhistorique s'est établie au croisement de la science archéologique (donc de l'histoire) et d'une soif métaphysique. Il a montré aussi que Bataille s'était tenu jusqu'au bout à ce croisement, sans occulter ni minorer aucun des deux horizons, et que l'importance de son Lascaux et la naissance de l'art venait de l'épreuve empathique qu'il avait faite de son objet d'étude, devenu intérieur au sujet écrivant. Du coup, forcément, la préhistoire de Bataille ne peut être que subjective : une préhistoire qui, pour lui, trouve son axe dans le symbolisme supposé de la fameuse "scène du puits » de Lascaux (où un homme schématique et ithyphallique tombe symboliquement face à un bison maternel aux entrailles pendantes, lui-même transpercé de flèches), pour formuler un désir à jamais interdit d'union avec la mère ; une préhistoire qui valorise donc le tragique de la séparation plutôt que la jouissance fusionnelle, l'expiation plutôt que la réconciliation, la fascination de l'inceste plutôt que la gloire de la parturition; une préhistoire qui, à la question de savoir quelle est la clé de l'énigme, "répond sans hésiter que c'est la mort» (cité p. 113), en dépit du «vitalisme foncier» de son inventeur (p. 98). La préhistoire selon Bataille (et selon Fabre) n'est ni douce ni rassurante ; elle se donne la violence et la mort comme noyau. Sauf qu'ultimement ce fond de hantises personnelles, indispensable à la montée poétique de la pensée, consent à se résoudre en non-savoir - ce que Fabre désigne comme une "involution vers l'enfance" (p. 70) ou une attraction vers "le silence " (titre de la dernière partie de son livre). C'est là, une fois de plus, que se loge la force spécifique de l'idée préhistorique : celle d'interdire que la grille d'aucun discours se referme sur l'antre des questions et d'imposer que la caverne ornée demeure un lieu pour toujours énigmatique où, écrit l'auteur de Bataille à Lascaux, "la lumière de l'origine, indéfiniment répétée pour chaque vivant, est comme fossilisée dans de merveilleuses images, en attente de régénération sous les regards les plus intenses et les plus affectés » (p. 122). 


\section{AUTEURS}

\section{RÉMI LABRUSSE}

remi.labrusse@wanadoo.fr 